\title{
ASYMPTOTIC FORMS FOR LAGUERRE POLYNOMIALS
}

\section{BENJAMIN MUCKENHOUPT ${ }^{1}$}

1. Introduction. In [2] Erdélyi proved two asymptotic estimates for Laguerre polynomials that are uniform in $n$ and $x$. Unfortunately, he proved these estimates only for $\alpha \geqq 0$ while Laguerre polynomials are of interest for all $\alpha>-1$. These estimates were used in [1], [4] and [5] to prove mean convergence theorems for Laguerre series; because of the restriction on $\alpha$ the result in [1] was proved only for $\alpha \geqq 0$ while in [4] and [5] some consequences of Erdélyi's estimates had to be proved separately for $-1<\alpha<0$.

The purpose of this paper is to show that the restriction in the estimates to $\alpha \geqq 0$ is unnecessary and that they are valid for all $\alpha>-1$. The method of proof is to use Erdélyi's estimates for positive $\alpha$ and some identities for Laguerre polynomials and Bessel functions. The proof works almost as well for $\alpha \leqq-1$ but some complications arise; since the results are of limited interest, they are not discussed here.

The Laguerre polynomials, $L_{n}^{\alpha}(x)$ are defined by

$$
\sum L_{n}^{\alpha}(x) r^{n}=(1-r)^{-\alpha-1} \exp \left(\frac{-r x}{1-r}\right) .
$$

Erdélyi's estimates are the following:

(1.1) Given $\alpha \geqq 0$ and $b<1$, there exists $n_{0}$ such that if $n \geqq n_{0}$ and $0<x \leqq v \nu$, then

$$
L_{n}^{\alpha}(x)=\frac{\Gamma(n+\alpha+1) 2^{\alpha-1 / 2} e^{x / 2} \psi^{1 / 2}}{n ! \nu^{\alpha / 2-1 / 2} x^{\alpha / 2+1 / 2}\left(\psi^{\prime}\right)^{1 / 2}}\left[J_{\alpha}(\nu \psi)+O\left(\frac{x^{1 / 2}}{\nu^{3 / 2}} \tilde{J}_{\alpha}(\nu \psi)\right)\right] .
$$

(1.2) Given $\alpha \geqq 0$ and $a>0$, there exists $n_{0}$ such that if $n \geqq n_{0}$ and $x \geqq a \nu$, then

$$
L_{n}^{\alpha}(x)=\frac{(-1)^{n} \pi^{1 / 2} 2^{5 / 6} N^{N+1 / 6} e^{x / 2}}{n !\left(-\phi^{\prime}\right)^{1 / 2} x^{\alpha / 2+1 / 2} e^{N}}\left[A i\left(-\nu^{2 / 3} \phi\right)+O\left(\frac{\widetilde{A i}\left(-\nu^{2 / 3} \phi\right)}{x}\right)\right] .
$$

In (1.1) and (1.2) $\nu=4 N=4 n+2 \alpha+2, t=x / \nu$,

Received by the editors May 7, 1969.

${ }^{1}$ Supported in part by N. S. F. grant GP 7539. 


$$
\begin{aligned}
\psi=\psi(t) & =\frac{1}{2}\left(t-t^{2}\right)^{1 / 2}+\frac{1}{2} \sin ^{-1} t^{1 / 2}, \\
\phi=\phi(t) & =[3 \beta(t) / 2]^{2 / 3}, \quad 0<t \leqq 1, \\
& =-[3 \gamma(t) / 2]^{2 / 3}, \quad 1<t, \\
\beta(t) & =\frac{1}{2} \cos ^{-1} t^{1 / 2}-\frac{1}{2}\left(t-t^{2}\right)^{1 / 2} \\
\gamma(t) & =\frac{1}{2}\left(t^{2}-t\right)^{1 / 2}-\frac{1}{2} \cosh ^{-1} t^{1 / 2}, \\
\tilde{J}_{\alpha}(u) & =u^{\alpha}, \quad 0<u \leqq 1, \\
& =u^{-1 / 2}, \quad 1<u, \\
\tilde{A} i(u) & =A i(u), \quad u \geqq 0, \\
& =\left[|A i(u)|^{2}+|B i(u)|^{2}\right]^{1 / 2}, \quad u<0,
\end{aligned}
$$

$J_{\alpha}$ is the Bessel function of order $\alpha$ as in [7] and $A_{i}$ and $B_{i}$ are Airy integrals as in [3].

The result of this paper can be stated as follows.

Theorem. Statements (1.1) and (1.2) are true if " $\alpha \geqq 0$ " is replaced " $\alpha>-1 "$.

2. Preliminary results. Three Laguerre polynomial identities will be needed; they are

and

$$
\begin{aligned}
L_{n}^{\alpha}(x) & =L_{n}^{\alpha+1}(x)-L_{n-1}^{\alpha+1}(x), \\
L_{n-1}^{\alpha+1}(x) & =-\frac{n}{x} L_{n}^{\alpha}(x)+\frac{n+\alpha}{x} L_{n-1}^{\alpha}(x)
\end{aligned}
$$

$$
L_{n}^{\alpha}(x)=\frac{\alpha+1-x}{n} L_{n-1}^{\alpha+1}(x)-\frac{x}{n} L_{n-2}^{\alpha+2}(x) .
$$

These are respectively (5.1.13), the second half of (5.1.14) and a combination of (5.1.2) and the first half of (5.1.14), pp. 99 and 101 of [6]. (2.1) will also be used in the form

$$
L_{n}^{\alpha}(x)=L_{n}^{\alpha-1}(x)+L_{n-1}^{\alpha}(x),
$$

and (2.2) will also be used in the form

$$
L_{n}^{\alpha}(x)=\frac{x}{n+\alpha+1} L_{n}^{\alpha+1}(x)+\frac{n+1}{n+\alpha+1} L_{n+1}^{\alpha}(x) .
$$

It will be necessary to have an expression for $L_{n}^{\alpha}(x)$ in terms of polynomials with larger $\alpha$ but the same value of $\nu=4 n+2 \alpha+2$. This will be useful since the estimates in (1.1) and (1.2) can then be substituted and most of the complicated functions will be the same in each term. In this derivation and the next, "use formula A to replace B" 
will mean "adjust $\alpha$ and $n$ in formula $\mathrm{A}$ so that the left side equals $\mathrm{B}$ and replace $B$ with the right side of this modified formula $A$ ".

To obtain the desired Laguerre polynomial expression, start with (2.3), use (2.1) to replace $L_{n-1}^{\alpha+1}(x)$ and then use (2.5) to replace $L_{n-2}^{\alpha+2}(x)$. This produces the identity

$$
\begin{array}{r}
L_{n}^{\alpha}(x)=\left[\frac{(\alpha+1)(\alpha+2)}{n(n+\alpha+1)}-\frac{x}{n}\right] L_{n-1}^{\alpha+2}(x) \\
-\frac{(\alpha+1) x}{n(n+\alpha+1)} L_{n-2}^{\alpha+3}(x) .
\end{array}
$$

Next, use (2.4) to replace the $L_{n+1}^{\alpha}(x)$ in (2.5); solving for $L_{n}^{\alpha}(x)$ produces

$$
L_{n}^{\alpha}(x)=\frac{x}{\alpha} L_{n}^{\alpha+1}(x)+\frac{n+1}{\alpha} L_{n+1}^{\alpha-1}(x) .
$$

Now use (2.7) to replace $L_{n-2}^{\alpha+3}(x)$ in (2.6). Combining terms produces the desired identity,

$$
\begin{aligned}
L_{n}^{\alpha}(x)= & {\left[\frac{(\alpha+1)(\alpha+2)}{n(n+\alpha+1)}-\frac{(\alpha+2)(2 n+\alpha+1) x}{n(n+\alpha+1)(\alpha+3)}\right] L_{n-1}^{\alpha+2}(x) } \\
& -\frac{(\alpha+1) x^{2} L_{n-2}^{\alpha+4}(x)}{n(n+\alpha+1)(\alpha+3)} .
\end{aligned}
$$

The following facts about Bessel functions will be needed. From (8), p. 40 of [7] and (1), p. 199 of [7] it follows immediately that for $u>0$

$$
\left|J_{\alpha}(u)\right| \leqq C \tilde{J}_{\alpha}(u)
$$

where $C$ depends only on $\alpha$. (1), p. 45 of [7] states that

$$
J_{\alpha}(u)=\frac{u}{2 \alpha}\left[J_{\alpha-1}(u)+J_{\alpha+1}(u)\right] .
$$

Now replace $\alpha$ by $\alpha+2$ in (2.10) and then use (2.10) to replace $J_{\alpha+1}(u)$ and $J_{\alpha+3}(u)$. Solving for $J_{\alpha}(u)$ in the resulting identity shows that for $\alpha \neq-3$

$$
\begin{aligned}
J_{\alpha}(u)= & {\left[\frac{-2(\alpha+2)}{\alpha+3}+\frac{4(\alpha+1)(\alpha+2)}{u^{2}}\right] J_{\alpha+2}(u) } \\
& -\frac{\alpha+1}{\alpha+3} J_{\alpha+4}(u) .
\end{aligned}
$$


The only fact needed about $A i(u)$ is that for all real $u$

$$
|A i(u)| \leqq \tilde{A} i(u) \text {; }
$$

(i) p. 510 and (20) p. 511 of [3] imply that $A i(u) \geqq 0$ for $u \geqq 0$, and, therefore imply (2.12) for $u \geqq 0$. (2.12) for $u<0$ follows immediately from the definition of $\tilde{A} i$.

By inspection of its definition $\psi(t)=t^{1 / 2}(1+O(t))$. Using this shows that

$$
\nu \psi=(\nu x)^{1 / 2}+O\left(x^{3 / 2} \nu^{-1 / 2}\right)
$$

and, since $\psi(t)$ has a positive lower bound for $0<\alpha \leqq t \leqq 1$,

$$
(\nu x)^{-1}=(\nu \psi)^{-2}+O\left(\nu^{-2}\right) .
$$

3. Proof of the theorem. Fix $\alpha$ and $b$ satisfying $-1<\alpha<0$ and $0<b<1$ and define

$$
p=\frac{\Gamma(n+\alpha+1) 2^{\alpha-1 / 2} e^{x / 2} \psi^{1 / 2}}{n ! \nu^{\alpha / 2-1 / 2} x^{\alpha / 2+1 / 2}\left(\psi^{\prime}\right)^{1 / 2}} ;
$$

$p$ is everything in the approximation (1.1) except for the bracket containing the Bessel function. Substituting the approximations in (1.1) for $L_{n-1}^{\alpha+2}(x)$ and $L_{n-2}^{\alpha+4}(x)$ into (2.8) shows that for $0<x \leqq b \nu$ and $n$ sufficiently large $L_{n}^{\alpha}(x)$ is equal to the sum of

$$
\begin{aligned}
p\left[\frac{4(\alpha+1)(\alpha+2)}{\nu x}-\frac{2(\alpha+2)}{\alpha+3}\right] \\
\cdot\left[J_{\alpha+2}(\nu \psi)+O\left(\frac{x^{1 / 2}}{\nu^{3 / 2}} \tilde{J}_{\alpha+2}(\nu \psi)\right)\right]
\end{aligned}
$$

and

$$
\begin{aligned}
& p\left[\frac{-16(n+\alpha+2)(n-1)(\alpha+1)}{(\alpha+3) \nu^{2}}\right] \\
& \cdot\left[J_{\alpha+4}(\nu \psi)+o\left(\frac{x^{1 / 2}}{\nu^{3 / 2}} \tilde{J}_{\alpha+4}(\nu \psi)\right)\right] .
\end{aligned}
$$

In the principal term of (3.2) replace the first $(\nu x)^{-1}$ by using (2.14) and in (3.3) write $(n+\alpha+2)(n-1)=\nu^{2} / 16+O(1)$. Adding (3.2) and (3.3) and using (2.11) and (2.9) shows that

$$
\begin{aligned}
L_{n}^{\alpha}(x)=p\left[J_{\alpha}(\nu \psi)+O\left(\frac{1}{\nu^{2}}+\frac{x^{1 / 2}}{\nu^{3 / 2}}+\frac{1}{x^{1 / 2} \nu^{5 / 2}}\right)\right. \\
\left.\cdot\left(\tilde{J}_{\alpha+2}(\nu \psi)+\tilde{J}_{\alpha+4}(\nu \psi)\right)\right] .
\end{aligned}
$$


Using the definition of $\tilde{J}_{\alpha},(2.13)$, and the fact that $0<x<\nu$, shows that the error term in (3.4) is

$$
O\left[\frac{p x^{\frac{1}{2}}}{\nu^{3 / 2}} \tilde{J}_{\alpha}(\nu \psi)\right] .
$$

This completes the proof of $(1.1)$ for $-1<\alpha<0$.

Next, fix $a>0, \alpha$ satisfying $-1<\alpha<0$ and define

$$
q=\frac{(-1)^{n} \pi^{\frac{1}{2}} 2^{5 / 6} N^{N+1 / 6} e^{\frac{1}{2} x}}{n !\left(-\phi^{\prime}\right)^{\frac{1}{2}} x^{\frac{1}{2} \alpha+\frac{1}{2}} e^{N}}
$$

$q$ is everything in the approximation (1.2) except for the bracket containing the Airy integral. Substituting the approximations in (1.2) for $L_{n-1}^{\alpha+2}(x)$ and $L_{n-2}^{\alpha+4}(x)$ into (2.8) shows that for $x \geqq a \nu$ and $n$ sufficiently large

$$
\begin{aligned}
L_{n}^{\alpha}(x)= & q\left[1-\frac{(\alpha+1)(\alpha+2)}{x(n+\alpha+1)}\right] \\
& \cdot\left[A i\left(-\nu^{2 / 3} \phi\right)+O\left(\frac{\widetilde{A i}\left(-\nu^{2 / 3} \phi\right)}{x}\right)\right] .
\end{aligned}
$$

An application of (2.12) will how produce the estimate for this $\alpha$. This completes the proof of the theorem.

\section{REFERENCES}

1. R. Askey and S. Wainger, Mean convergence of expansions in Laguerre and Hermite series, Amer. J. Math. 87 (1965), 695-708. MR 32 \#316.

2. A. Erdélyi, Asymptotic forms for Laguerre polynomials, J. Indian Math. Soc. 24 (1960), 235-250. MR 23 \#A1073.

3. H. Jeffreys and B. S. Jeffreys, Methods of mathematical physics, 3rd ed., Cambridge Univ. Press, Cambridge, 1956. MR 17, 590.

4. B. Muckenhoupt, Mean convergence of Hermite and Laguerre series. I, Trans. Amer. Math. Soc. (to appear).

5. - Mean convergence of Hermite and Laguerre series. II, Trans. Amer. Math. Soc. (to appear).

6. G. Szegö, Orthogonal polynomials, rev. ed., Amer. Math. Soc. Colloq. Publ. vol. 23, Amer. Math. Soc., Providence, R. I., 1959. MR 21 \#5029.

7. G. Watson, A treatise on the theory of Bessel functions, 2nd ed., Cambridge Univ. Press, Cambridge, 1966.

Rutgers University and

The Institute for Advanced Study 